\title{
Doppler measurement of cardiac output during cardiopulmonary resuscitation
}

\author{
D I Fodden, A C Crosby, K S Channer
}

\begin{abstract}
Objective-To estimate the cardiac output produced by external cardiac compression during standard cardiopulmonary resuscitation performed by two groups of operators with different levels of experience and training.

Methods-Cardiac output was measured by Doppler aortovelography. All patients included in the study had necropsy examinations. Only patients without evidence of pulmonary embolism, myocardial rupture, aortic valve disease, or acute depletion of the intravascular volume were included.

Results-31 patients presenting to the accident and emergency department suffering from non-traumatic cardiac arrest had cardiac output measurements made during resuscitation. Eleven patients were excluded after necropsy examination. The median cardiac index for the 20 study patients was $3.2 \mathrm{~L} \mathrm{~min} \mathrm{~mm}^{-1} \mathrm{~m}^{-2}$. The cardiac output produced by massage by less experienced personnel (median $1.2 \mathrm{~L} \mathrm{~min}^{-1} \mathrm{~m}^{-2}$ ) was significantly less than that produced by those fully trained in the technique (median $3.2 \mathrm{~L} \mathrm{~min}^{-1} \mathrm{~m}^{-2}$; $\mathbf{P}<0.0195 \%$ confidence interval -2.36 to -1.29$)$. The amount of resuscitation related trauma was no greater than in other published series.

Conclusions-Differences in cardiac output during external cardiac compression are related to experience with the technique.

$(\mathcal{A}$ Accid Emerg Med 1996;13:379-382)
\end{abstract}

Key terms: cardiac output; cardiopulmonary resuscitation; Doppler aortovelography

Royal Hallamshire

Hospital, Glossop

Road, Sheffield,

United Kingdom:

Accident and

Emergency

D I Fodden

A C Crosby

Cardiology

K S Channer

Correspondence to: Dr K S Channer, Cardiology Department, Royal Department, Royal Trust, Glossop Road, Sheffield S10 2JF

Accepted for publication 8 May 1996 plunger and pneumatic vest have been developed. ${ }^{7}$ Estimation of cardiac output following these modifications has largely been by invasive methods.
Cardiac output can be estimated noninvasively using Doppler aortovelography. ${ }^{9}$ In this technique, a beam of high frequency sound is reflected back from moving blood cells. The frequency of the reflected sound varies with the velocity and direction of the target. ${ }^{10}$ From the difference in the frequency of the incident and reflected beams and the cross sectional area of the ascending aorta, the cardiac output can be estimated. In most cases the cross sectional area of the aorta cannot be accurately measured, so alternative Doppler indices of cardiac output have been used including systolic velocity integral or stroke distance. These Doppler indices have been shown to reflect cardiac output. ${ }^{11}$ In this study, necropsy examinations after unsuccessful resuscitation allowed measurement of aortic diameter so that cardiac output could be assessed more accurately.

The aim of our study was to compare the cardiac output produced by external cardiac compression when this was performed by personnel with different levels of experience and training. We also compared the degree of resuscitation trauma associated with different operators.

\section{Methods}

PATIENTS

Patients who had suffered a non-traumatic cardiorespiratory arrest were eligible for the study. Standard cardiopulmonary resuscitation was carried out in accordance with the Resuscitation Council guidelines. ${ }^{12}$ The patients were intubated and ventilated with $100 \%$ oxygen by hand using a Water's circuit. External cardiac compression was performed at a rate of $80 / \mathrm{min}$ and the lungs inflated after every fifth compression. The massage was performed by one of the three personnel, each of whom had exceeded the criteria for a pass result of the Resuscitation Examination for Membership of the Royal College of General Practitioners. ${ }^{13}$ In the case of a cardiorespiratory arrest, it is accepted practice to rotate personnel to perform external cardiac massage and this minimises operator fatigue. In order to assess whether the cardiac output was operator dependent, additional measurements were made on seven cases, with the massage being performed by less experienced operators. These personnel (student nurses) had been trained in basic life support and were familiar with the technique, having used mannequins previously, but were not greatly experienced in cardiac massage on humans. The measurements of cardiac output were made after those of the skilled personnel. 
Table 1 Necropsy diagnoses

\begin{tabular}{cccl}
\hline Case & Sex & Age & Diagnosis \\
\hline 1 & M & 71 & Myocardial infarction, fractured ribs \\
2 & M & 59 & Myocardial ischaemia, hypertension, aspiration of gastric contents \\
3 & M & 46 & Myocardial infarction, fractured ribs \\
4 & F & 71 & Overdose of tricyclic antidepressants \\
5 & F & 67 & Subarachnoid haemorrhage \\
6 & M & 85 & Myocardial infarction, fractured ribs \\
7 & M & 77 & Septicaemia, peritonitis, fractured ribs \\
8 & M & 52 & Epileptic, vomited, aspiration of gastric contents \\
9 & M & 58 & Myocardial infarction, hypertension \\
10 & F & 79 & Myocardial infarction, fractured ribs \\
11 & M & 56 & Myocardial infarction \\
12 & M & 65 & Congestive heart failure, myocardial ischaemia, hypertension \\
13 & F & 22 & Refractory ventricular fibrillation, myocardial fibrosis \\
14 & M & 51 & Myocardial infarction, fractured sternum \\
15 & M & 65 & Myocardial ischaemia, hypertension, fractured ribs \\
16 & M & 63 & Myocardial infarction \\
17 & M & 15 & Refractory ventricular fibrillation, hypoxia, solvent abuse \\
18 & F & 64 & Left ventricular failure, myocardial ischaemia, hypertension \\
19 & M & 63 & Congestive heart failure, recent myocardial infarction, fractured ribs \\
20 & M & 66 & Myocardial ischaemia, fractured sternum \\
\hline
\end{tabular}

\section{CARDIAC OUTPUT}

Cardiac output was estimated using Doppler aortovelography (Sci Med-PC Dop 842). The aortic flow velocity was estimated according to the formula:

$$
v=\delta f \times c / 2 f \times \cos (\theta),
$$

where $\mathrm{v}=$ magnitude of blood flow velocity; $\mathrm{df}=$ measured change in sound frequency; $\mathrm{c}=$ velocity of sound in tissue; $f=$ frequency of emitted Doppler signal; $\theta=$ angle between the direction of blood flow and the Doppler signal

The value of $\theta$ is zero because the direction of the Doppler beam is directly along the ascending aorta. This is facilitated by the use of a specifically angled $2 \mathrm{MHZ}$ pulsed Doppler probe which produces a wide beam. The mean Doppler shift frequency is extracted from the Doppler spectrum which is displayed in real time. Mean velocity is obtained from the first moment of the Doppler power spectrum, which is computed automatically. Cardiac output is then obtained from the product of the time averaged mean velocity and cross sectional area of the aorta. This technique produces reproducible results. We measured the coefficient of variation for 10 repeated measurements on the same subject on 10 occasions by the same experienced observer and found the mean cardiac output was $4.25 \mathrm{~L}$ $\mathrm{min}^{-1}$, the standard deviation was $0.36 \mathrm{~L} \mathrm{~min}^{-1}$, and the coefficient of variation was $8.5 \%$.

The measurements of cardiac output were made by the same person (DIF). The probe was placed in the suprasternal notch, using a standard coupling gel, and the Doppler beam was directed along the ascending aorta. The orientation of the probe was manoeuvred to produce the best waveform on the computer screen. The loudspeaker used to provide the Doppler audio signal was switched off and the computer screen which displayed the Doppler signal visually was out of sight of the operator performing the massage in order to remove any feedback which might have affected the technique used. A $600 \mathrm{~Hz}$ wall filter was applied to remove high energy signals from movement of the aortic wall ("wall thump"), and depth settings were adjusted to place the sample volume in the central part of the ascending aorta about
$3 \mathrm{~cm}$ above the valve, where the anatomy is more constant. Each measure of cardiac output averages data from 15 to 20 cardiac cycles and 10 measurements of cardiac output were made for each subject.

To calculate the cardiac output, the computer software requires a value for the aortic root diameter; it was therefore programmed with a value of $30 \mathrm{~mm}$ and the data were saved on to the computer disk. When the actual root diameter was known from a necropsy examination, the data were retrieved from disc and amended in order to recalculate the cardiac output. It is these recalculated measures of cardiac output that are quoted in the results section.

The Doppler measurements were made independently of the medical staff involved with the resuscitation, and the decision to terminate resuscitation was made on clinical grounds; the presence of the additional equipment was irrelevant in this respect.

\section{NECROPSY EXAMINATION}

Necropsy examination of the patients was undertaken, and if the myocardium or circulatory system was not intact, if there was evidence of major antemortem haemorrhage, if pulmonary thromboembolism was present, or if there was any evidence of aortic valvular stenosis or incompetence, the case was excluded from the study. The aorta was split longitudinally and the internal circumference measured at a distance of three $\mathrm{cm}$ above the aortic valve leaflets and parallel to their plane. From this measurement, the aortic root diameter was calculated and this value was then fed back into the Doppler computer for calculation of the actual cardiac output. The length and weight of the body were measured at necropsy and from these the body surface area ${ }^{14}$ and the cardiac index were calculated.

\section{STATISTICAL METHODS}

The results of the cardiac output produced by the two groups of operators were compared using the Mann-Whitney $U$ test.

\section{Results}

Thirty one cases were assessed. Eleven were excluded because necropsy examination was not undertaken or because it showed one of the exclusion criteria, so 20 cases were entered into the study. In each case, basic cardiac life support was begun within five minutes of the cardiac arrest and measurement of cardiac output begun within 20 minutes. The mean age of the study group was 59.7 years and there were 16 women and 15 men. The necropsy diagnoses are shown in table 1 along with details of the resuscitation related trauma.

\section{CARDIAC OUTPUT}

There was no evidence of deterioration of cardiac output throughout the 10 recordings while cardiac massage was performed by the fully proficient personnel. The median cardiac output and cardiac index values were $5.8 \mathrm{~L}$ $\mathrm{min}^{-1} \mathrm{~m}^{-2}$ and $3.2 \mathrm{~L} \mathrm{~min}^{-1} \mathrm{~m}^{-2}$ respectively, and the individual results of each case are shown in table 2 . 
Table 2 Median and range of cardiac output and cardiac index in 20 cases

\begin{tabular}{|c|c|c|}
\hline Case & $\begin{array}{l}\text { Cardiac output } \\
\left(\text { min }^{-1}\right)\end{array}$ & $\begin{array}{l}\text { Cardiac index } \\
\left(\mathrm{Lin}^{-1} \mathrm{~m}^{-2}\right)\end{array}$ \\
\hline 1 & 7.3 & 3.5 \\
\hline 2 & 6.8 & 3.3 \\
\hline 3 & 6.4 & 3.2 \\
\hline 4 & 4.9 & 2.7 \\
\hline 5 & 6.2 & 3.4 \\
\hline 6 & 5.4 & 2.7 \\
\hline 7 & 5.5 & 3.0 \\
\hline 8 & 4.5 & 2.9 \\
\hline 9 & 3.9 & 2.6 \\
\hline 10 & 6.1 & 3.3 \\
\hline 11 & 4.5 & 3.2 \\
\hline 12 & 4.6 & 2.6 \\
\hline 13 & 5.2 & 3.1 \\
\hline 14 & 4.9 & 2.6 \\
\hline 15 & 6.0 & 3.5 \\
\hline 16 & 6.8 & 3.4 \\
\hline 17 & 6.5 & 3.3 \\
\hline 18 & 5.1 & 2.5 \\
\hline 19 & 5.8 & 3.3 \\
\hline 20 & 6.6 & 3.4 \\
\hline Median (range) & $5.8(3.9-7.3)$ & $3.2(2.5-3.5)$ \\
\hline
\end{tabular}

Overall, there were seven cases in which ribs were fractured and a further two in which the sternum was fractured. The median cardiac index produced by proficient personnel in subjects with fractures of the chest wall was $3.3 \mathrm{~L}$ $\mathrm{min}^{-1} \mathrm{~m}^{-2}$ and that in subjects without any fractures was $3.1 \mathrm{~L} \mathrm{~min}^{-1} \mathrm{~m}^{-2}$. This difference was not significant (Mann-Whitney U test, 95\% confidence interval -0.11 to 0.57 ).

The results of the cardiac output produced by less skilled operators are shown in table 3 . There was no difference in the cause of death compared with those cases whose cardiac massage was performed entirely by more skilled personnel. Of the seven cases in which novices performed cardiopulmonary resuscitation, three $(43 \%)$ had a fracture of the chest wall, compared with six of the $13(46 \%)$ in whom cardiopulmonary resuscitation was performed solely by experienced operators ( $P=N S$, Exact test). The median cardiac index produced by the "novice" operators was $1.2 \mathrm{~L} \mathrm{~min}^{-1} \mathrm{~m}^{-2}$, while that of the "proficient" operators on the same subgroup was $3.2 \mathrm{~L} \mathrm{~min}^{-1} \mathrm{~m}^{-2}(P<0.01$, Mann-Whitney U test, $95 \%$ confidence interval -2.36 to -1.29 ).

\section{Discussion}

We have shown that the cardiac output produced by cardiopulmonary resuscitation is dependent upon the skill of the operator. Even if the cardiac output measured by our technique is inaccurate, comparative data from the same subject are valid since the potential sources of error are the same. Although novice operators always followed experienced operators, we do not think that the lower measures of cardiac output which they produced can be explained by clinical deterioration of the patients because the change in cardiac output produced by the first and second operators was large and we had not observed any downward trend in the cardiac output with repeated measurements with the same skilled operator.

We have shown that the skill of a competent operator maximises cardiac output without increasing injury to the chest wall or to the
Table 3 Median and range of cardiac index $\left(\mathrm{L} \mathrm{min}^{-1} \mathrm{~m}^{-2}\right)$ producd by external cardiac compression by novice and proficient operators

\begin{tabular}{lll}
\hline Case & Novice & Proficient \\
\hline 3 & 1.2 & 3.2 \\
8 & 1.9 & 2.9 \\
10 & 1.9 & 3.3 \\
11 & 0.9 & 3.2 \\
16 & 1.3 & 3.4 \\
18 & 0.8 & 2.5 \\
20 & 1.1 & 3.4 \\
Median (range) & $1.2(0.8-1.9)$ & $3.2(2.5-3.4)$ \\
\hline
\end{tabular}

thoracic or abdominal contents. The cardiac output produced in the canine model increases significantly with a modest increase in the depth of compression ${ }^{14}$ but the incidence of resuscitation related trauma in our series of 20 cases was no greater than in other published series. ${ }^{15}$ It is possible that the enhanced cardiac output which we have shown is due to more vigorous external cardiac compression, but without an increase in trauma.

The normal resting cardiac index is 2.6 to $3.6 \mathrm{~L} \mathrm{~min}^{-1} \mathrm{~m}^{-217}$ and we have shown that the cardiac output produced by cardiopulmonary resuscitation can be equivalent to that measured in life. We have used a non-invasive (Doppler) technique to measure cardiac output and this has been shown to correlate well with both thermodilution $^{18}$ and indicator dilution techniques. ${ }^{19}$ Although cardiopulmonary resuscitation will produce movement of the chest wall and heart, the Doppler probe was placed in the suprasternal notch, remote from the area of largest movement, and visual images of the Doppler output were similar to those obtained in life. We cannot, however, exclude the possibility that movement artefact may have increased the Doppler signal, producing an increased estimate of cardiac output. Certainly, previous data have shown that the cardiac output during cardiopulmonary resuscitation is up to $42 \%$ of the normal resting cardiac output, the measurements having been made in the canine or porcine model. ${ }^{20}{ }^{21}$ The techniques which have been used include electromagnetic flowmeters, thoracic electrical bioimpedance, dilution techniques, and Doppler assessment of the velocity of carotid artery blood flow. ${ }^{19} 22{ }^{23}$ More recently, studies have been undertaken measuring end tidal carbon dioxide during cardiopulmonary resuscitation and this reflects pulmonary blood flow. ${ }^{24} 25$

The outcome of resuscitative efforts depends upon several factors including the cause of the arrest, the time delay to the onset of basic life support, and the distribution of blood flow during resuscitation. The effort of cardiopulmonary resuscitation is to maintain adequate cerebral and cardiac oxygenation until spontaneous cardiac output returns; the long term outcome is therefore dependent upon cerebral and cardiac blood flow. While we have shown that a normal cardiac output can be produced by external cardiac compression, we have made no effort to assess the distribution of the cardiac output. In the situation of external cardiac compression, the blood flow is directed predominantly cephalad. ${ }^{21}$ Smaller Doppler probes would be required to assess blood flow 
along the major arteries branching from the aortic arch to verify this distribution of flow.

The Royal College of General Practitioners has recently introduced a practical assessment of a candidates competence to provide basic cardiac life support. The examination requires that at least $70 \%$ of the compressions satisfy strict criteria of hand position, sternal compression, and rate of compression when assessed on a mannequin. ${ }^{13}$ Our experienced personnel have satisfied more strict criteria, which required the hand position to be correct for $100 \%$ of the compressions and that at least $85 \%$ of the compressions, tested on a mannequin, were correct with regard to both the depth and rate of sternal compression. It is likely, therefore, that the higher cardiac output produced by our experienced personnel reflects better training. We have subsequently used the Doppler technique to feedback directly to the operator in order to maximise cardiac output during cardiopulmonary resuscitation in humans.

In conclusion, we have shown that the cardiac output, measured by Doppler aortovelography - produced when fully trained personnel use standard cardiopulmonary resuscitation - can be similar to the resting cardiac output. The cardiac output produced is operator dependent, being higher when massage is performed by fully trained personnel. Previous research has been invasive or used animal models, and the Doppler technique which we have used is simple to use and reliable. It can be used to give direct feedback to the masseur in order to help maximise cardiac output from cardiopulmonary resuscitation.

1 Kouwenhoven WB, Jude JR, Knickerbocker GG. Closedchest cardiac massage. $\mathscr{f} A M A$ 1960;173:1064-7.

2 Cohn JD, Del Guercio LRM. Cardiorespiratory analysis of cardiac arrest and resuscitation Surg Gymecol Obstet 1966;123:1066-70.

3 Berryman CR, Phillips GM. Interposed abdominal compression-CPR in human subjects. Ann Emerg Med compression-CPR

4 Fitzgerald KR, Babbs CF, Frissora HA, Davis RW, Silver

Fitzgerald KR, Babbs CF, Frissora HA, Davis RW, Silver
DI. Cardiac output during cardiopulmonary resuscitation DI. Cardiac output during cardiopulmonary resuscitation
at various compression rates and durations. Am $¥$ Physiol at various compressio.
5 Chandra N, Rudikoff M, Weisfeldt ML. Simultaneous ches compression and ventilation at high airway pressure during cardiopulmonary resuscitation. Lancet 1980;i:175-8.

6 Babbs CF, Tucker WA, Paris RL, Murphy RJ, Davis RW. CPR with simultaneous compression and ventilation at high airway pressure in 4 animal models. Crit Care Med 1982;10:501-4.

7 Cohen TJ, Tucker KJ, Lurie KG, Redberg RF, Dutton JP, Dwyer KA, et al. Active compression-decompression. A new method of cardiopulmonary resuscitation. $\mathfrak{F} A M A$ new method of 2 .

8 Halperin HR, Tsitlik JE, Gelfand M, Weisfeldt ML, Gruben KG, Levin HR, et al. A preliminary study of cardiopulmonary resuscitation by circumferential compression of the nary resuscitation by circumferential compression of the chest with the

9 Light $\mathrm{H}$. Transcutaneous aortovelography. A new window on the circulation? Br Heart $\mathcal{F}$ 1976;38:433-42.

10 Atkinson P, Woodcock JP. Doppler ultrasound and its use in clinical measurements. London: Academic Press, 1982.

1 Rawles JM. Stroke distance, an improved measure of cardiovascular function. Scott Med f 1989;34:394-8.

12 Evans TR,ed. $A B C$ of resuscitation. London: BMJ Publishing Group, 1990.

13 The Royal College of General Practitioners. Cardiopulmonary resuscitation performance test. London: The Royal College of General Practitioners, 1991.

14 Du Bois D, Du Bois EF. A formula to estimate the approximate surface area if height and weight be known. Arch Intern Med 1916;17:863-71.

15 Babbs CF, Vorrhees WID, Fitzerald KR, Holmes HR, Geddes LA. Relationship of blood pressure and flow during CPR to chest compression amplitude: evidence for an CPR to chest compression amplitude: evidence for an effective

16 Krischer JP, Fine EG, Davis JH, Nagel EL. Complications of cardiac resuscitation. Chest 1987;92:287-91.

17 Stefan J, Gregora Z, Krumlov AV. Traumatic complications of indirect cardiac massage. Soudni Lekarstvi 1989;34:4953.

18 Wintrobe MM, Thorn GW, Adams RD, Braunwald E, Isselbacher KJ, Petersdorf RG, eds. Harrison's principles of internal medicine. New York: McGraw-Hill, 1974.

19 Distante A, Moscarelli E, Rovai D, L'Abbate A. Monitoring of change in cardiac output by transcutaneous aortovelography, a non-invasive Doppler technique: comparison with thermodilution. $₹$ Nucl Med Sci 1980;24:171-5.

20 Evans JM, Skidmore R, Baker JD, Wells PNT. A new approach to the non-invasive measurement of cardiac outapproach to the non-invasive measurement of cardiac out-
put using an annular array Doppler technique. II. Practical put using an annular array Doppler technique. II. Practical impl.

21 Silver DI, Murphy RJ, Babbs CF, Geddes LA. Cardiac output during CPR: a comparison of two methods. Crit Care Med 1981;9:419-20.

22 Sharff JA, Pantley G, Noel E. Effect of time on regional organ perfusion during two methods of cardiopulmonary resuscitation. Ann Emerg Med 1984;13:649-56.

23 Lopez-Saucedo A, Hirt M, Appel PL, Curtis DL, Harrie HD, Shoemaker WC. Feasibility of non-invasive physiologic monitoring in resuscitation of trauma patients in the emergency department. Crit Care Med 1989;17:567-8.

24 Maier GW, Tyson GS, Olsen CO, Kernstien KH, Davis JW, Conn EH, et al. The physiology of external cardiac massage; high-impulse cardiopulmonary resuscitation. Circulation 1984;70:86-101.

25 Ward KR, Sullivan RI, Zelenak RR, Summer WR. A comparison of interposed abdominal compression CPR and standard CPR by monitoring end-tidal $\mathrm{PCO}_{2}$. Ann Emerg Med 1989;18:831-7.

26 Weil MH, Bisera J, Trevino RP, Rackow EC. Cardiac output and end-tidal carbon dioxide. Crit Care Med 1985;13: 907-9. 